\title{
Anthropomorphic Information Systems
}

\author{
Nicolas Pfeuffer • Alexander Benlian • \\ Henner Gimpel • Oliver Hinz
}

Received: 17 November 2017/ Accepted: 2 October 2018/Published online: 27 May 2019

(c) Springer Fachmedien Wiesbaden GmbH, ein Teil von Springer Nature 2019

Keywords Anthropomorphic technology

Anthropomorphic information systems · Anthropomorphic design - Conversational agents · Human-computer interaction

\section{Anthropomorphism in Information Systems}

Anthropomorphism is the attribution of human-like physical or non-physical features, behavior, emotions, characteristics and attributes to a non-human agent or to an inanimate object (Epley et al. 2007). Anthropomorphism as a human innate tendency has been well documented for a long time in the history of humanity. Early drawings about 30,000 years ago depict animals with human-like bodies (Dalton 2004). The main goal of the projection of humanlike attributes onto non-human agents is to facilitate the

Accepted after one revision by Prof. Dr. Weinhardt.

N. Pfeuffer · Prof. Dr. O. Hinz ( $\square)$

Information Systems and Information Management, Goethe

University Frankfurt, Frankfurt am Main, Germany

e-mail: ohinz@wiwi.uni-frankfurt.de

N. Pfeuffer

e-mail: pfeuffer@wiwi.uni-frankfurt.de

Prof. Dr. A. Benlian

Information Systems and E-Services, Technical University

Darmstadt, Darmstadt, Germany

e-mail: benlian@ise.tu-darmstadt.de

Prof. Dr. H. Gimpel

Research Center Finance and Information Management,

University of Augsburg, Augsburg, Germany

e-mail: henner.gimpel@fim-rc.de understanding and explanation of the behavior and intentions of the non-human agents (Epley et al. 2007).

Nass et al. (1996) were among the first to provide experimental evidence that humans perceive computers in an anthropomorphic way. In their research on the "computers are social actors" paradigm, they found that humans tend to apply social heuristics for interactions with computers that are imbued with human or social cues. The social interaction with the machines exposed a seemingly unnatural attribution of human characteristics to the computers, which not only lead to socially correct manners towards the inanimate objects, such as politeness (Nass et al. 1999), but it also led to emotional and positive reactions towards computers (de Melo et al. 2014; Nass et al. 1996). This kind of behavior can be attributed to anthropomorphism.

Anthropomorphism at the human-computer interface is usually triggered by anthropomorphic cues within information technology (IT). Since anthropomorphism constitutes an opportunity to influence the users of IT, software and hardware developers aim to apply anthropomorphic features and designs to give humans a familiar feeling with IT because a natural and personal connection to a piece of hard- or software is missing. This anthropomorphic design invokes anthropomorphism which makes it easier for humans to connect with the system and therefore facilitates the familiarization with its features (Burgoon et al. 2000; Epley et al. 2007).

Anthropomorphic features which emerge from anthropomorphic design are manifold and include, for example, voice recognition as well as voice synthesizing and computer-graphical rendering of human-like faces or bodies, including mimics and gestures. Yet anthropomorphism is not only about addressing the visual or auditory aspects of the interaction in a more human way, but also about the 
contents in that interaction. Recent advancements in machine learning (ML) and natural language processing (NLP) techniques have enabled chatbots, for example, to behave more naturally and give more contextually sensitive responses, thus providing semantically correct answers and more trustworthy experiences in interactions which in turn increases their human-likeness (Abdul-Kader and Woods 2015; Li et al. 2016). More readily available examples of such artefacts that include anthropomorphic features are virtual assistants such as Google Home and Amazon's Alexa that can serve as some form of butler in a smart home.

One of the primary goals of anthropomorphic design is to positively influence humans' affect, which is observed to be an important factor in human-computer interaction (HCI) (Hudlicka 2003; Ochs et al. 2017). Imbuing systems or robots with anthropomorphic cues may have many positive implications, such as higher likeability due to social or emotional connection (Eyssel et al. 2010), an increase of decision quality in purchase processes because of a more natural interaction (Qiu and Benbasat 2009) or even improvements in sociability of children diagnosed with autism spectrum conditions (Bernardini et al. 2014). Yet, anthropomorphic design might also have negative effects when the technology cannot deliver on the expectations it creates, as discussed in literature on the "uncanny valley" (Mori 1970; Saygin et al. 2012). Furthermore, the discrepancy between the user goals, the expectations and actual capabilities of those systems, often referred to as the "gulfs of execution and evaluation" (Norman 1986), sometimes lead to amusement and confusion among users. Especially if those systems are imbued with anthropomorphic features, negative emotions such as frustration or even aggression could be nurtured by these gulfs, which may lead to a loss of trust in those systems (Norman 1986). Consequently, this would decrease the credibility of these intelligent systems as well as the usage intention and finally create a loss of economic value for the operating companies.

Therefore, it is an important goal of information systems research to deepen the insights into the influence and acceptance of anthropomorphism in information systems to leverage their full potential.

This article first introduces the term 'anthropomorphism', prevailing theories about this term as well as its connection to information systems. Next, we propose a categorization of anthropomorphic features and show previous research in information systems on these features, including acceptance and influence of anthropomorphic features on the users. Finally, we point out future challenges for anthropomorphic information systems research.

\section{Anthropomorphism}

\subsection{A Conceptualization of Anthropomorphism}

The phenomenon of anthropomorphism has been discussed by scholars of various disciplines such as social psychology, personality psychology, and anthropology since several centuries (Darwin 1998/1873; Epley et al. 2007; Feuerbach 2004/1873; Freud 1930). Basically, anthropomorphism describes the attribution of human-like physical or non-physical features, behavior, emotions, characteristics and attributes to a non-human agent or to an inanimate object (Epley et al. 2007). The application of anthropomorphism towards a non-human agent or entity occurs particularly when logical knowledge about a non-human agent is missing. In these kinds of situations, the motivation to socialize and to understand the non-human agent may lead to the application of anthropocentric knowledge. This can be attributed to the subconscious intention to better understand events or circumstances containing nonhuman agents as well as to understand the non-human agents themselves. Without applying anthropomorphism, said events or non-human agents would not be logically explainable or understandable by the human agent.

The inductive inference process of anthropomorphism resembles the process of cognitive biases such as anchoring or overconfidence, in the manner that human agents use the most readily available information - the information about humans - to make a final conclusion about a non-human agent (Epley 2004; Epley et al. 2007; Griffin and Tversky 1992). Epley et al. (2007) predict that especially people who have little time or little cognitive resources to form an induction are more likely to form a final judgement that is biased by readily available anthropocentric knowledge (Epley et al. 2007).

Epley et al. (2007) further contend that cognitive as well as motivational aspects of humans determine the likelihood of using anthropocentric knowledge to form a conclusion about a non-human agent or an inanimate object. The theory they developed to explain the application of anthropomorphism contains three main factors which influence the likelihood of a human applying anthropomorphism:

1. Elicited agent knowledge is a key determinant to explain the behavior or properties of a non-human agent. If a human agent has acquired none or little knowledge about a non-human agent, the human agent is likely to use the readily available information about human behavior or human properties to form a judgement. When a human agent collects more information about the non-human agent, the acquired information should activate alternate knowledge 
structures that compete with the knowledge about humans. This in turn should lead to a correction of the judgement about the non-human agent and therefore decrease the likelihood and degree of anthropomorphism.

2. Effectance in anthropomorphism is derived from the effectance motivation introduced by White (1959) and describes the motivation of a human agent to understand and interact effectively with a non-human agent. By applying human characteristics and attributing human intentions to non-human agents, the human agent aims to better understand the motivation behind the non-human agent's actions and reduce the uncertainty about the non-human agent's future actions. Therefore, anthropomorphism helps human agents to reduce uncertainty, anxiety and to keep a situation under control.

3. Anthropomorphism also serves the goal of sociality motivation, which is the need to connect to other human beings. By establishing a social connection with the non-human agent, the goal of sociality motivation is satisfied. Conversely, this means that if a human lacks a social connection to other humans he or she is more likely to anthropomorphize.

Epley et al. (2007) argue that these three factors interplay as follows: Elicited agent knowledge determines if and how a human agent will use anthropomorphism as a source of knowledge in the inductive reasoning process to form a judgement about a non-human agent. The inductive reasoning process, however, is also influenced by sociality and effectance motivation.

In the case of little elicited agent knowledge, a high need of a social connection, which means an increased sociality motivation, for example, increases the ease of accessibility for anthropomorphic representations of nonhuman agents and will therefore lead to a higher probability of anthropomorphizing. Similarly, high levels of effectance motivation also lead to a higher probability of anthropomorphizing.

Effectance motivation will reinforce the usage of alternate knowledge structures to better understand non-human agents, if non-anthropocentric knowledge about the nonhuman agent is available. In contrast, even if non-anthropocentric knowledge about a non-human agent is available, sociality motivation will increase the will to anthropomorphize nevertheless (Epley et al. 2007).

\subsection{Anthropomorphic Design}

Since humans are used to attribute human-like characteristics as well as emotions to inanimate objects or nonhuman agents from the early childhood on (Derby 1970;
Lanier Jr et al. 2013), it is no surprise that consumer research as well as product marketers have discovered anthropomorphism as a design pattern for products which are more likeable to human beings (Aggarwal and McGill 2007; Landwehr et al. 2011; Wen Wan et al. 2017). Landwehr et al. (2011), for example, identified the significant effect of cellphones purposely designed in an anthropomorphic way calling to mind the eyes and mouth in a human face. Their results indicate that by choosing a thoughtful design for the right parts of a product, the consumer will anthropomorphize. As a result, consumers may like the product better.

Positive effects of anthropomorphic design have also been observed in information technology and information systems. For example, in a study on product recommendation agents (PRA) in an e-commerce platform, Qiu and Benbasat (2009) found that an anthropomorphic design, especially anthropomorphized voices and embodiment of PRA, positively influenced social presence which in turn lead to a higher trust into and credibility of the technological agent's recommendations. These kinds of features can already be found in the design of current artificial agent designs by businesses. For example, minimal visual cues such as the blinking eyes of Anki's home assistant vector (Anki 2018) are used to enhance trustworthiness of the robot. Anthropomorphic embodiments can be found in recommendation agents such as noora, who helps customers to find a suitable spa in Switzerland (Paixon GmbH 2018). Moreover, human-like voices of Google's Assistant or Apple's Siri foster the social connection between the user and the agents and promote a more natural interaction and trust (Apple Inc. 2018; Google LLC 2018).

Anthropomorphic feature design has been a part of human-computer interaction for a long time and has provided several facets of novel interaction methods that facilitate the user's life and make it easier to connect with the technology (Burgoon et al. 2000). Consequently, technology industry players aim to equip their products with anthropomorphic features such as voice synthesizers, to enhance their products with a greater human touch (Mädche et al. 2016). On the other hand, anthropomorphic design of automated user assistance systems is an opportunity for companies of various industries to improve customer satisfaction and reduce their service costs at the same time (Gnewuch et al. 2017).

Applying anthropomorphic features to IT has shown to positively influence likeability. However, beyond a certain threshold, the level of human-likeness might have a negative effect as postulated by the "uncanny valley" (Fig. 1). Initially coined by Mori, the uncanny valley describes the area of a graph for likeability of a human-like object, in which the human-likeness of that object reaches a point where it creates an eerie feeling on the side of the human 
Fig. 1 Conceptualization of the uncanny valley as described by Mori (1970)

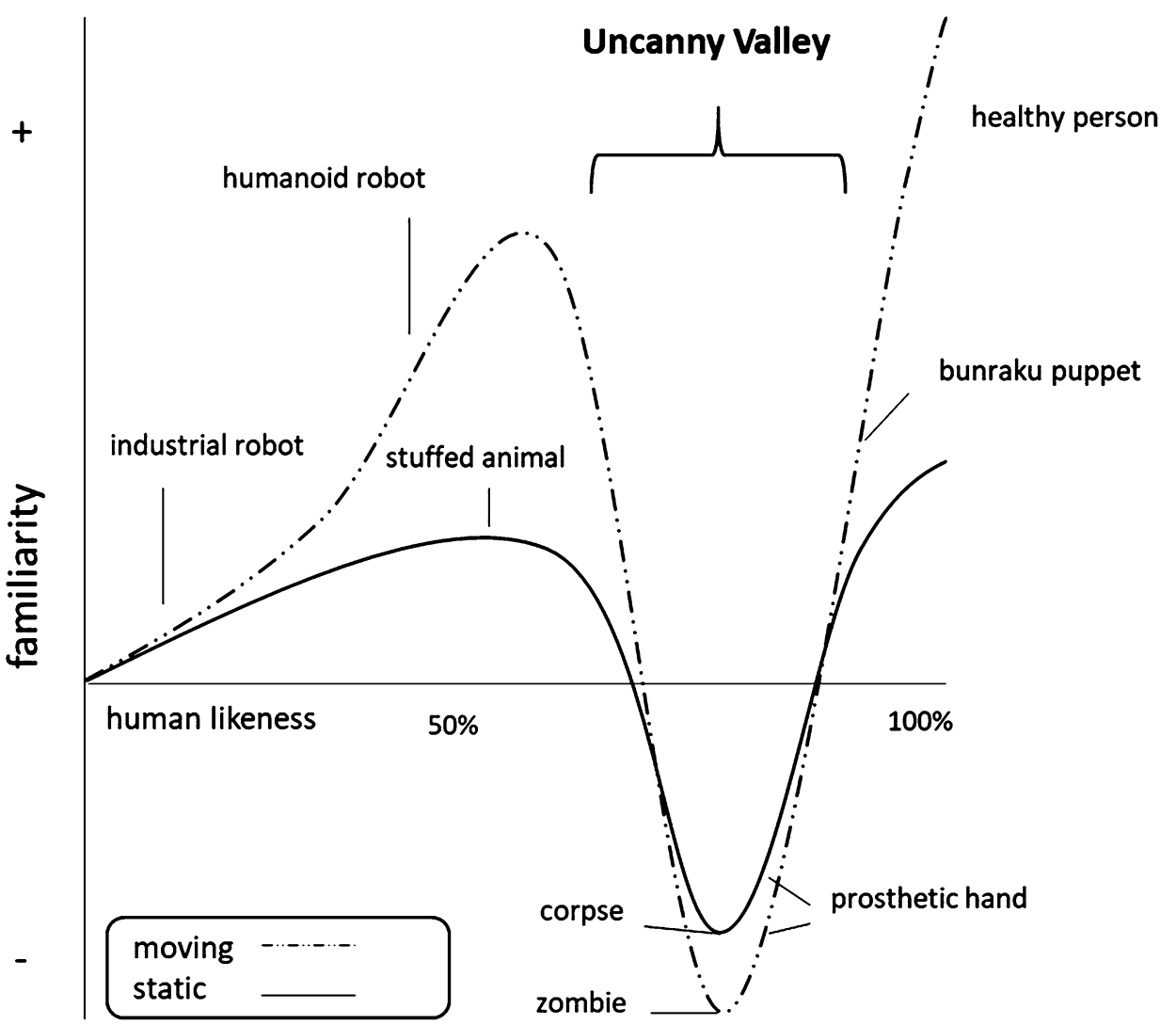

agent which replaces the initially high likeability (Mori 1970). The reason for this eeriness is that the anthropomorphic design creates expectations the anthropomorphic object cannot fulfil. Once the object reaches the next higher degree of human-likeness and transcends the uncanny valley, it is able to meet the expectations and is perceived as very human-like and thus the feeling of uncanniness disappears. Intelligent information systems, such as virtual assistants (Cortana, Alexa) or embodied conversational agents (Nunamaker et al. 2011) - which are becoming more and more human-like in behavior, intelligence, looks or motion - might hit the uncanny valley at a certain point. However, recent research on the uncanny valley has been debating the existence and the potential reasons for this effect (Bartneck et al. 2007; Burleigh et al. 2013).

\subsection{Anthropomorphic Information Systems}

Information systems (IS) are socio-technical systems comprised of technical, informational and social artefacts that are embedded in a technical language and in a social context (Iivari 2017; Lee et al. 2015). Anthropomorphic IS (AIS) are IS in which the technical and informational artefacts possess cues that tend to lead humans to attribute human-like physical or non-physical features, behavior, emotions, characteristics and attributes to the IS.
There are many possible conceptualizations of IS and not all explicitly point to the role of information artefacts. Following both Lee et al. (2015) and Iivari (2017), the explicit inclusion of the information artefact alongside (information) technology aims to take information seriously as object for analysis and design. This appears relevant to anthropomorphic IS in order to highlight that the anthropomorphic cues within the IS might be part of both the technical artefacts (e.g., an audio interface) and the informational artefacts (e.g., usage of human names). Like any IS, such anthropomorphic IS are in part designed and in part emergent (Iivari 2017).

The people interacting with anthropomorphic IS are not part of the IS itself but are users from the environment of the IS (Iivari 2017). They respond to the information system's anthropomorphic cues by anthropomorphizing the IS. Figure 2 shows the process of anthropomorphism within IS in accordance to our definition and the definition of anthropomorphism by Epley et al. (2007).

Anthropomorphic features in the design of IS, which include anthropomorphic technical artefacts and anthropomorphic information artefacts as well as their effects on the users of the IS, are manifold and therefore require a clear categorization. Our exemplary, but not exhaustive categorization of anthropomorphic features is based on the idea that the concept of anthropomorphism can be applied 


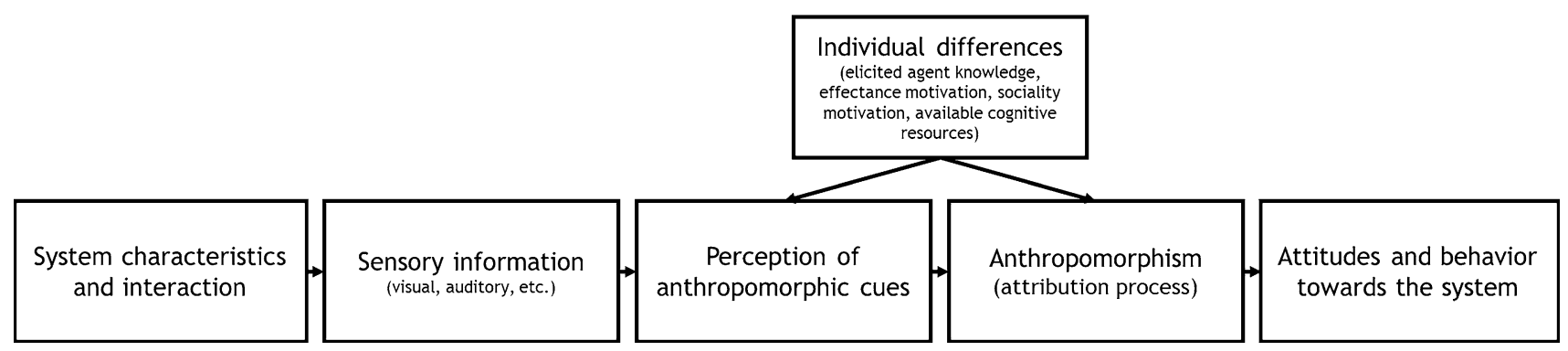

Fig. 2 Process of anthropomorphism in IS

Table 1 Categories of anthropomorphic features from a user perspective

\begin{tabular}{ll}
\hline $\begin{array}{l}\text { Categories of anthropomorphic features from } \\
\text { a user perspective }\end{array}$ & Exemplary manifestations of anthropomorphic features \\
\hline Visual & Appearance (Goetz et al. 2003; Kim et al. 2016; Landwehr et al. 2011) \\
& Movements (Saygin et al. 2012) \\
& Gestures (Bailenson and Yee 2005) \\
& Mimics (Beale and Creed 2009) \\
& Gender (Eyssel and Hegel 2012) \\
& Speech synthesizer (Abdul-Kader and Woods 2015; Eyssel et al. 2012; Nunamaker et al. 2011) \\
& Gender (Powers et al. 2005; Siegel et al. 2009) \\
Auditory & Cognitive intelligence (e.g., Dialog ability, context, content understanding, image processing, \\
& speech recognition) (Gnewuch et al. 2017; Hudlicka 2003; Nunamaker et al. 2011) \\
Mental (Cognitive, emotional, behavioral) & Emotionality and emotional intelligence (Beale and Creed 2009; Eyssel et al. 2010; Picard et al. \\
& 2001; Zhang et al. 2008) \\
& Personality (André and Rist 2001; Wang 2017)
\end{tabular}

if anthropomorphic elements were actively detected during the perception and cognition processes or induced via sociality or effectance motivation.

Based on our literature review in the research disciplines of information systems, human computer interaction, sociology and psychology, we could not find a clear categorization of anthropomorphic features. Therefore, we derived three broad categories that are recognizable from an anthropocentric perspective of mental processes that involve perception and cognition through sensory information (Epley et al. 2007; Nisbett and Wilson 1977). The three categories consist of elementary anthropomorphic features that imply humanity and can be grasped by the human senses, whereas in contrast to cyber-physical systems, AIS only require the senses of vision and hearing, as AIS are not necessarily physical. Table 1 depicts the categories of features and common manifestations of these features. Visual and auditory features can rather be perceived directly from sensory information. In contrast, the assessment of mental features requires a more cognitively complex inference process based on sensory information. An example for mental features is an information system showing signs of cognitive intelligence, for example, via context understanding, content understanding, and dialog ability (Luger and Sellen 2016; Schuetzler et al. 2014). For the user, this might be conveyed via the system's speech output (relating to an auditory anthropomorphic feature) or via text output on a screen. The latter is visual sensory input for the user but not a visual anthropomorphic feature itself. Typically, not the display of text on a screen will trigger anthropomorphism but the users' cognitive processing of this text when it leads to the perception, that the IS features cognitive intelligence. Anthropomorphic features like visual appearance (Eyssel and Hegel 2012) and voice (Powers et al. 2005; Siegel et al. 2009) may also lead to the perception of biological gender in an IS which in turn triggers the sense of behavioral, cultural, or psychological traits typically associated with either men or women. This demonstrates that the categories are distinct but interrelated. When using multiple anthropomorphic features, anthropomorphic design will typically aim to create a consistent perception like, for example, appearance and voice suggesting the same gender, displaying mouth movements when speech is uttered, or displaying gestures consistent with current emotionality. Thinking through the cognitive complexity of perceiving the anthropomorphic features and the interplay of multiple such features is an important part of anthropomorphic design in order to tailor 
the features to the intended users' general and contextspecific abilities and needs.

Visual features aim to improve the social connection to non-human technological agents by applying motoric and static human features to non-human agents. For example, an anthropomorphized appearance such as a human-like face on a virtual agent may improve the bond between the human and the virtual agent especially in interactions which are more social in nature, such as the interaction between an instructor and an apprentice (Broadbent et al. 2013; Goetz et al. 2003).

It is important to acknowledge that especially visual anthropomorphic cues can be conveyed both through software features, such as a smiling virtual agent, and through hardware features (Fig. 3), which can be observed in the design of the virtual assistant in the paper by Benlian et al. (2019).

Auditory features as anthropomorphic interaction methods such as speech synthesizers combined with mental features such as voice recognition can greatly improve the usefulness and ease of use of an IS or IT, especially for physically or visually impaired people, while also improving the perceived efficacy of those users (AbdulKader and Woods 2015). Furthermore, more intricate features such as gender may be conveyed through auditory features, which may influence the perception of skills or attributes of an artificial agent and therefore lead to different user behavior (Eyssel and Hegel 2012).

Finally, the perception of mental features such as emotional intelligence or personality is a complex process, which can be influenced by an array of factors. For example, embodied virtual agents could present emotions through visual cues such as a mimic resembling a smile (Fig. 4), which would result in the corresponding human agent to perceive the virtual agent as happy, which makes him more sociable (Eyssel et al. 2010). In contrast to the expression of emotions, features such as emotional or cognitive intelligence are more difficult to convey credibly, since those features require a thorough analysis of the

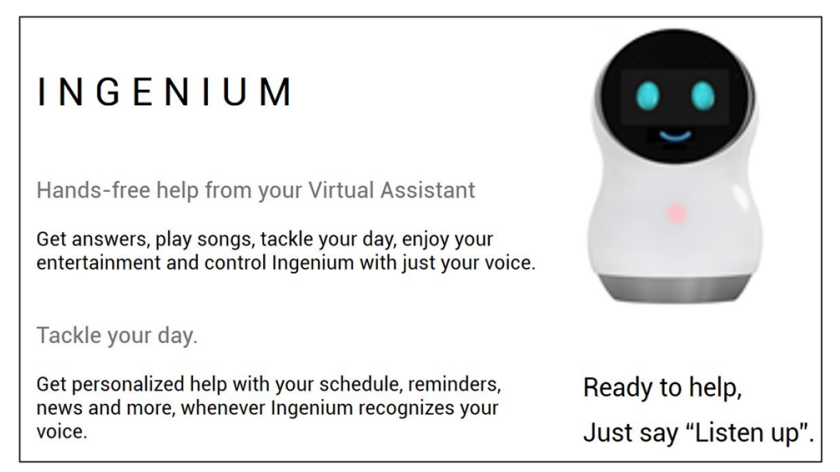

Fig. 3 An embodied virtual assistant with a smiling face (Benlian et al. 2019)
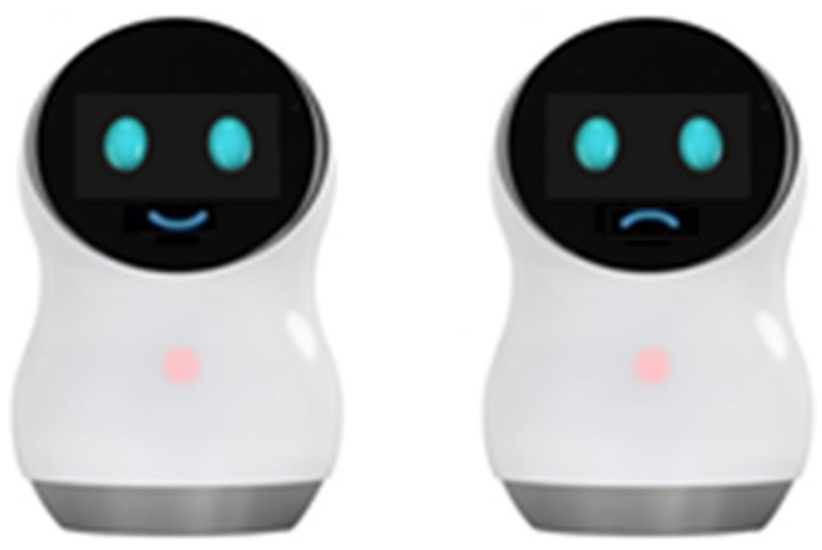

Fig. 4 Happy and sad embodied virtual agent (Benlian et al. 2019)

context and content of an interaction between a human agent and a non-human technological agent.

\section{Research Topics Concerning Anthropomorphism in Information Systems}

Literature that deals with anthropomorphic features is mostly situated in the area of human-computer interaction as well as computer science, yet only available to a limited extent in the domain of information systems research.

The research area within anthropomorphism that has probably attracted most attention in information systems is 'Conversational Agents'. Conversational IS that implement certain anthropomorphic features to provide a more natural interaction are categorized as Conversational Agents (CA) (Wilks 2010). CA can be regarded as a superclass for several other types of anthropomorphic IS, which will be discussed in further detail below (Luger and Sellen 2016). Table 2 provides an overview of topics of interest in this area that have emerged through the application of anthropomorphic features to information systems.

Since anthropomorphism comes into effect during the interaction with a non-human agent, human-computer interaction plays an integral role for anthropomorphizing information systems. During human-computer interaction, information systems converse through verbal or non-verbal means with the user to convey relevant information or to provide certain functionalities. At this point we want to clarify that the goal of Table 2 is to provide a schematic overview of topics of interest in the area of CA. Yet beyond the exemplary types of CA that we mention, there are several additional areas that have been examined by disciplines other than information systems. Among those areas, for example, teaching assistants or health assistants may be found (Elmasri and Maeder 2016; Heller et al. 2005). 
Table 2 Types of conversational agents

\begin{tabular}{llll}
\hline Main purpose & Exemplary types of CA & Areas of applicability & Literature \\
\hline $\begin{array}{llll}\text { Conversational } \\
\text { Conversion optimization }\end{array}$ & Social bot & Social media & Edwards et al. (2014) \\
& Anthropomorphized product & Marketing, advertising & Qiu and Benbasat (2009) \\
& $\begin{array}{l}\text { Recommendation agent (PRA) } \\
\text { Banking assistant }\end{array}$ & Financial industry & Jung et al. (2017, 2018) \\
& Customer service assistant & Customer service & Gnewuch et al. (2017) \\
Information delivery and personal & Game assistant & Gaming industry & Kim et al. (2016) \\
process optimization & User assistant/personal butler & Individual & Luger and Sellen (2016), \\
& & & Mädche et al. (2016) \\
& Smart home assistant/assisted living & Assisted living, & Mettler et al. (2017) \\
\hline
\end{tabular}

Chatbots were one of the earliest attempts of forming $\mathrm{CA}$ and enjoy great popularity today, which is why these terms are often used interchangeably. The implementation of chatbots requires natural language understanding/processing (NLU/NLP), which is used to converse with human agents through text-based chat in a natural verbal manner (Luger and Sellen 2016). Stemming from artificial intelligence, the idea behind and goal of chatbots was to create an artificially intelligent conversational system that appeared and acted so humanly through its conversational feature that interacting humans could not distinguish these chatbots from real humans. Early examples include the chatbots Eliza as well as the superior, although paranoid PARRY (Colby et al. 1971; Weizenbaum 1966). What rendered PARRY clearly superior to Eliza was that its messages seemed not only context-aware, but that its conversation strategy also revealed a paranoid attitude, which made him more human-like. Several decades later, the even more sophisticated Cleverbot deceived about 59\% of 1334 participants in a Turing Test experiment into thinking it was human (Aron 2011).

In a study involving Cleverbot, Hill et al. (2015) evaluated if humans act differently in instant messaging (IM) conversations if they know their opposite is a non-human agent. Their method included the comparative analysis of a sample of 100 human-human IM conversations and 100 human-chatbot conversations. One of their major findings was that humans adapt their conversation behavior to that of the chatbots by using fewer words per message, but more messages in general, to make it easier for the chatbots to process the input. Furthermore, an excessive use of profanity by the users within the human-chatbot conversations (30 times more compared to human-human conversations) revealed that humans were aware that they were talking to a non-human agent, but also explorative about the chatbot's capabilities to react accordingly (Hill et al. 2015).
Social bots are - in contrast to simpler chatbots - not mainly conversation-oriented, but fulfill various economic purposes. A study by Edwards et al. (2014), which surveyed the perception of messages by Twitterbots, suggests that human agents regard content delivered by social bots of organizations as a credible, reliable source of information. Thus, the integration of bots in social media provides a powerful tool for organizations and even governments to influence people within social networks, such as Facebook, Twitter and Instagram. For example, social bots with a large network have been suspected to have strongly influenced discussions in social media revolving around the presidential election of the U.S.A. in 2016 (Bessi and Ferrara 2017).

Also anthropomorphized product recommendation agents (PRA) exert influence on individuals, but rather in the sales context. This type of conversational agent engages with customers to satisfy both their needs for a fitting product and the aim of the online-shop to maximize profits. Here the PRA plays the role of a salesperson, which can greatly enhance customer satisfaction and loyalty by providing functional (advice, time savings, enhanced purchase decisions) and social (social relationship) benefits (Qiu and Benbasat 2009; Reynolds and Beatty 1999). The results of a study by Qiu and Benbasat (2009), for example, indicate that both visual (embodiment/appearance) and auditory (human voice synthesizer) features strongly influence social presence, which in turn directly influences trust and enjoyment, which may finally lead to a greater acceptance of the PRA among customers.

Similar to anthropomorphized PRA, banking assistants aim to replace the traditional role of a human advisor, i.e., the banking assistant, financial investment advisor or insurance advisor (Shah et al. 2017). In these roles, banking assistants can be responsible for managing fortune or assisting in making financial decisions. In the past years of the digitization of economy, the deployment of Robo-Advisors as non-human financial and insurance service agents 
has become more and more popular and is seen by several scholars as the second stage of digitization that enables the financial industry to target new customer segments (Jung et al. 2018). Jung et al. (2018) recently pointed out that few researchers in IS have approached the topic of Robo-Advisors. In their opinion Robo-Advisory provides rich opportunities for research that can enhance and establish guidelines for the design of Robo-Advisors. The authors highlight especially the topics User Interface Design, Customer Behavior and Risk measurement and modelling as important. Although Robo-Advisors are not directly associated with $\mathrm{CA}$, the implementation of a CA as a natural interface for Robo-Advisors not only seems natural, but looks like a promising research area.

Customer service assistants (CSA) undertake tasks that have been carried out by human employees in customer service in the past. As such, the tasks of CSA include handling customer complaints or queries on the phone or service counter tasks such as recommending movies in a movie theater (Gnewuch et al. 2017; Lee and Choi 2017). Although CSA have been in use for several years now, prospective customers often deem these systems as less useful than traditional customer service. Gnewuch et al. (2017) identified several issues that hinder the success of CSA, such as a limited understanding of natural language, missing context-awareness that leads to a lack in responsiveness as well as an overly human appearance and behavior that creates expectations the CSA cannot fulfil. The latter may be interpreted as an occurrence of the uncanny valley.

In a study on Game assistants - virtual embodied conversational agents, which guide and support video game players with hints and tips in a video game - Kim et al. (2016) observed that a seemingly too dominant assistant can even undermine the invocation of enjoyment, although the latter is the prime goal of a video game product.

In particular, because the game assistants conveyed a great deal of anthropomorphic features, their advices were perceived as too authoritarian, thus leading to the perception of the game assistants as a threat to the player's autonomy (Kim et al. 2016). The observations provided by Kim et al. (2016) highlight the fact that the unthoughtful application of anthropomorphic feature design without a thorough understanding of the invoked effects could lead to negative outcomes.

User assistant/personal butler (UAS) systems, such as Microsoft's Cortana, Apple's Siri and Google's Google Now have become increasingly important in the everyday life of smartphone users (Luger and Sellen 2016). The primary goal of those systems is to convey information in real-time to a user and enrich information systems by their ability to support the user's cognitive capabilities with their social interface and technical functions (Mädche et al.
2016). Although a variety of intelligent UAS are available on the market, Mädche et al. (2016) deem their features and HCI capabilities as insufficient. They argue that, for achieving the superior goal of truly intelligent UAS which they call "advanced UAS", UAS should not exclusively provide intelligence or an interactive experience, but proactive, adaptive, context-aware and need-oriented experience.

Finally, assistants integrated into a smart home environment are not only beneficial for users who want to efficiently organize their domestic environment, but they could also provide a valuable opportunity for the healthcare and insurance industry; foremost, the European commission is running a dedicated ambient and assisted living program (AAL), which supports technological contributions that reduce costs of health and social care for elderly people. Under the impression that literature on voice-based smart home assistants as well as literature on AAL assistants is somewhat scarce, we find that the study and implementation of a primitive chat-based AAL assistant by Mettler et al. (2017) provides a valuable starting point for the design of AAL assistants, although both functionality and anthropomorphic features are limited.

\section{Outlook and Research Agenda}

This catchword aimed at emphasizing the relevance of the emerging research topic of anthropomorphism for IS research. Looking at previous research, it becomes evident that several design questions still need to be addressed and different application areas of anthropomorphized IS may have different presuppositions for an effective design, acceptance and usage. However, research for all types of anthropomorphized IS needs to answer common important questions.

Therefore, we call upon the members of the BISE community to come up with purposeful theoretical knowledge and especially design theories for an efficient and effective anthropomorphic IS design. To better target the specific expertise of the BISE members, we compiled a preliminary research agenda for different departments of the BISE journal. Based on our findings, Table 3 poses central research questions according to the different research departments within the BISE journal, which can help to identify further research questions.

All in all, 17 research questions emerged for five directions of research, which make the relevance of AIS for research become apparent. What especially gained our attention during our literature review, beyond the questions within Table 3, was that research on anthropomorphic technology mostly focused on novel engineering 
Table 3 Suggested topics for a research agenda for the BISE departments

\begin{tabular}{ll}
\hline Departments & Topics \\
\hline Decision analytics and data science & How should the models of AIS be designed such that they are not only cognitively intelligent, but \\
also emotionally intelligent to support the user in the best way possible? & Which machine learning approaches are the most promising in training models that enable \\
anthropomorphic features? & What kind of training data is necessary to train models for anthropomorphic features? \\
What are the best methods for mining and/or generating suitable training data? \\
What relevant business information can be gained from the user interactions with AIS, especially \\
concerning conversational agents?
\end{tabular}

Economics of information systems

Enterprise modeling and enterprise IS

Information systems engineering and technology (IS engineering)

Management and use of information and knowledge
How can we (statistically) determine the likelihood and strength of the appearance of anthropomorphism in the HCI context?

How do AIS affect customer relationship management and revenue?

How do anthropomorphic features affect user acceptance and trust in the IS?

How do anthropomorphic features influence human behavior and decision-making?

What underlying processes and mediation mechanisms (e.g., based on attribution, self-regulation or theories) drive the effect of anthropomorphic features on human decision-making?

What characteristics of the user context and human-computer interaction context (i.e., moderating effects) shape the influence of anthropomorphic features on human decisionmaking?

What are valuable application scenarios for AIS within enterprises?

How does the application of AIS affect the collaboration between human entities and organization units?

Which conversational models for conversational agents perform best for different platforms and industries: generative, selective or hybrid models?

How can anthropomorphic IS such as conversational agents improve the quality of service of enterprise information systems?

What impact do AIS have on work morale, productivity, work efficiency and satisfaction of employees?

What are best practices to integrate AIS or anthropomorphic features into an existing enterprise system landscape? techniques as well as HCI research on robots, yet not so much on interaction and design aspects of anthropomorphized IS.

Furthermore, while research in computer science have paid substantial attention to the interaction aspect in anthropomorphic information technologies, researchers have neglected the social science perspective for too long. Especially previous research disregarded several systematic differences between certain user groups, such as age, computer efficacy, sociality motivation, personality type or culture, which are important factors regarding the interaction with virtual non-human agents. We believe that through the inclusion of established IS constructs, a clear image of the acceptance of anthropomorphic features could be fostered and better insights into the factors that influence human-computer interaction can be gained.

We expect that without thorough research the gulfs of evaluation and execution will hold back an effective usage and wide adoption of anthropomorphized IS. To point out a particular example, this means that the duality of anthropomorphized non-human agents and human agents in the service context will remain. The proposal of preliminary meta-requirements and design principles for conversational agents in customer service by Gnewuch et al. (2017) could assist to overcome the obstacles of CSA design.

Nevertheless, (1) more research on the general understanding of anthropomorphism in IS needs to be conducted, to be able to (2) correctly assess the effects of features and human-likeness on human agents and (3) to evolve new design methodologies that can act as guidelines for the design of sound anthropomorphic IS.

We believe that thoughtfully designed anthropomorphic IS, such as conversational agents, are the key to greater acceptance, usefulness, usability, enjoyment of IS as well as a better user and customer experience and higher satisfaction with services. With the immense potential impact of anthropomorphic IS as well as the various ambitious and yet unsolved - research tasks at the intersection of information technology engineering and social sciences, anthropomorphic information systems provide an exciting research opportunity for the BISE community. 


\section{References}

Abdul-Kader SA, Woods J (2015) Survey on chatbot design techniques in speech conversation systems. Int $\mathbf{J}$ Adv Comput Sci Appl 6(7):72-80

Aggarwal P, McGill AL (2007) Is that car smiling at me? Schema congruity as a basis for evaluating anthropomorphized products. J Consum Res 34(4):468-479

André E, Rist T (2001) Presenting through performing: on the use of multiple lifelike characters in knowledge-based presentation systems. Knowl-Based Syst 14(1-2):3-13

Anki (2018) Anki vector: the home robot with interactive AI technology. Anki USA. https://www.anki.com/en-us/vector. Accessed 30 Oct 2018

Apple Inc. (2018) Siri. https://www.apple.com/uk/siri/. Accessed 30 Oct 2018

Aron J (2011) Software tricks people into thinking it is human. New scientist. https://www.newscientist.com/article/dn20865-softwaretricks-people-into-thinking-it-is-human/. Accessed 13 Feb 2018

Bailenson JN, Yee N (2005) Digital chameleons: automatic assimilation of nonverbal gestures in immersive virtual environments. Psychol Sci 16(10):814-819

Bartneck C, Kanda T, Ishiguro H, Hagita N (2007) Is the uncanny valley an uncanny cliff? In: 16th IEEE international symposium on robot and human interactive communication. IEEE Service Center, Piscataway, pp 368-373

Beale R, Creed C (2009) Affective interaction: how emotional agents affect users. Int J Hum Comput Stud 67(9):755-776

Benlian A, Klumpe J, Hinz O (2019) Mitigating the intrusive effects of smart home assistants by using anthropomorphic design features: a multi-method investigation. Inf Syst J (forthcoming). Preprint version available: https://www.researchgate.net/publica tion/332187512_Mitigating_the_Intrusive_Effects_of_Smart_ Home_Assistants_by_using_Anthropomorphic_Design_Fea tures_A_Multi-Method_Investigation

Bernardini S, Porayska-Pomsta K, Smith TJ (2014) ECHOES: an intelligent serious game for fostering social communication in children with autism. Inf Sci 264:41-60

Bessi A, Ferrara E (2017) Social bots distort the 2016 US presidential election online discussion. First Monday. https://doi.org/10. 5210/fm.v21i11.7090

Broadbent E, Kumar V, Li X, Sollers JS 3rd, Stafford RQ, MacDonald BA, Wegner DM (2013) Robots with display screens: a robot with a more humanlike face display is perceived to have more mind and a better personality. PLoS ONE 8(8):e72589. https://doi.org/10.1371/journal.pone.0072589

Burgoon JK, Bonito JA, Bengtsson B, Cederberg C, Lundeberg M, Allspach L (2000) Interactivity in human-computer interaction: a study of credibility, understanding, and influence. Comput Hum Behav 16(6):553-574

Burleigh TJ, Schoenherr JR, Lacroix GL (2013) Does the uncanny valley exist? An empirical test of the relationship between eeriness and the human likeness of digitally created faces. Comput Hum Behav 29(3):759-771. https://doi.org/10.1016/j. chb.2012.11.021

Colby KM, Weber S, Hilf FD (1971) Artificial paranoia. Artif Intell 2(1): $1-25$

Dalton R (2004) Lion man takes pride of place as oldest statue. Nature. https://doi.org/10.1038/425007a

Darwin C (1998/1873) The expression of the emotions in man and animals. Oxford University Press, Oxford (Original work published in 1873)

de Melo CM, Carnevale PJ, Read SJ, Gratch J (2014) Reading people's minds from emotion expressions in interdependent decision making. J Pers Soc Psychol 106(1):73-88
Derby J (1970) Anthropomorphism in children's literature or “Mom, my doll's talking again”. Elem Engl 47(2):190-192

Edwards C, Edwards A, Spence PR, Shelton AK (2014) Is that a bot running the social media feed? Testing the differences in perceptions of communication quality for a human agent and a bot agent on twitter. Comput Hum Behav 33:372-376

Elmasri D, Maeder A (2016) A conversational agent for an online mental health intervention. In: Proceedings of the international conference on brain and health informatics. Springer, Cham, pp 243-251

Epley N (2004) A tale of tuned decks? Anchoring as accessibility and anchoring as adjustment. In: Koehler DJ, Harvey N (eds) The Blackwell handbook of judgment and decision making. Blackwell, Malden, pp 240-256

Epley N, Waytz A, Cacioppo JT (2007) On seeing human: a threefactor theory of anthropomorphism. Psychol Rev 114(4):864-886

Eyssel F, Hegel F (2012) (S)he's got the look: gender stereotyping of robots. J Appl Soc Psychol 42(9):2213-2230

Eyssel F, Hegel F, Horstmann G, Wagner C (2010) Anthropomorphic inferences from emotional nonverbal cues: a case study. In: Proceedings of the IEEE international conference on robot and human interactive communication. IEEE, Viareggio, pp 646-651

Eyssel F, Kuchenbrandt D, Bobinger S, Ruiter L de, Hegel F (2012) 'If you sound like me, you must be more human'. In: Proceedings of the 7th annual ACM/IEEE international conference on human-robot interaction. IEEE, Boston, pp 125

Feuerbach L (2004/1873) The essence of religion. Prometheus, Amherst (Original work published in 1873)

Freud S (1930) Civilization and its discontents. Norton, New York, pp 64-145

Gnewuch U, Morana S, Mädche A (2017) Towards designing cooperative and social conversational agents for customer service. In: Proceedings of the international conference on information systems. AIS, Seoul

Goetz J, Kiesler S, Powers A (2003) Matching robot appearance and behavior to tasks to improve human-robot cooperation. In: Proceedings of the 12th IEEE international workshop on robot and human interactive communication. IEEE, Milbrae, pp 55-60

Google LLC (2018) Google Assistant-your own personal Google. https://assistant.google.com/. Accessed 30 Oct 2018

Griffin D, Tversky A (1992) The weighing of evidence and the determinants of confidence. Cogn Psychol 24(3):411-435

Heller B, Proctor M, Mah D, Jewell L, Cheung B (2005) Freudbot: an investigation of chatbot technology in distance education. In: World conference on educational media and technology. AACE, Montreal, pp 3913-3918

Hill J, Ford WR, Farreras IG (2015) Real conversations with artificial intelligence: a comparison between human-human online conversations and human-chatbot conversations. Comput Hum Behav 49:245-250

Hudlicka E (2003) To feel or not to feel: the role of affect in humancomputer interaction. Int J Hum Comput Stud 59(1):1-32

Iivari J (2017) Information system artefact or information system application: that is the question. Inf Syst J 27(6):753-774

Jung D, Dorner V, Weinhardt C, Pusmaz H (2017) Designing a roboadvisor for risk-averse, low-budget consumers. Electron Mark 28(3):367-380

Jung D, Dorner V, Glaser F, Morana S (2018) Robo-advisory. Bus Inf Syst Eng 60(1):81-86

Kim S, Chen RP, Zhang K (2016) Anthropomorphized helpers undermine autonomy and enjoyment in computer games. J Consum Res 43(2):282-302

Landwehr JR, McGill AL, Herrmann A (2011) It's got the look: the effect of friendly and aggressive "facial" expressions on product liking and sales. J Mark 75(3):132-146 
Lanier CD Jr, Rader CS, Fowler AR III (2013) Anthropomorphism, marketing relationships, and consumption worth in the toy story trilogy. J Mark Manag 29(1-2):26-47

Lee S, Choi J (2017) Enhancing user experience with conversational agent for movie recommendation: effects of self-disclosure and reciprocity. Int J Hum Comput Stud 103:95-105

Lee AS, Thomas M, Baskerville RL (2015) Going back to basics in design science: from the information technology artifact to the information systems artifact. Inf Syst J 25(1):5-21

Li J, Monroe W, Ritter A, Galley M, Gao J, Jurafsky D (2016) Deep reinforcement learning for dialogue generation. In: Proceedings of the 2016 conference on empirical methods in natural language processing, association for computational linguistics, Austin, pp 1192-1202

Luger E, Sellen A (2016) "Like having a really bad PA": the gulf between user expectation and experience of conversational agents. In: Proceedings of the 2016 CHI conference on human factors in computing systems. ACM, San Jose, pp 5286-5297

Mädche A, Morana S, Schacht S, Werth D, Krumeich J (2016) Advanced user assistance systems. Bus Inf Syst Eng 58(5):367-370

Mettler T, Bächle M, Daurer S, Judt A (2017) Parental control reversed: using ADR for designing a low-cost monitoring system for elderly. In: Proceedings of the international conference on information systems. AIS, Seoul

Mori M (1970) The uncanny valley. Energy 7(4):33-35

Nass C, Fogg BJ, Moon Y (1996) Can computers be teammates? Int J Hum Comput Stud 45(6):669-678

Nass C, Moon Y, Carney P (1999) Are people polite to computers? Responses to computer-based interviewing systems. J Appl Soc Psychol 29(5):1093-1109

Nisbett RE, Wilson TD (1977) Telling more than we can know: verbal reports on mental processes. Psychol Rev 84(3):231

Norman DA (1986) Cognitive engineering. In: Norman DA, Draper SW (eds) User-centered system design: new perspectives on human-computer interaction. Lawrence Erlbaum, Hillsdale, pp 31-61

Nunamaker JF, Derrick DC, Elkins AC, Burgoon JK, Patton MW (2011) Embodied conversational agent-based kiosk for automated interviewing. J Manag Inf Syst 28(1):17-48

Ochs M, Pelachaud C, Mckeown G (2017) A user perception - based approach to create smiling embodied conversational agents. ACM Trans Interact Intell Syst 7(1):4

Paixon GmbH (2018) Willkommen beim Wellness-Ratgeber Noora.ch. https://noora.ch/chat. Accessed 30 Oct 2018

Picard RW, Vyzas E, Healey J (2001) Toward machine emotional intelligence: analysis of affective physiological state. IEEE Trans Pattern Anal Mach Intell 23(10):1175-1191
Powers A, Kramer AD, Lim S, Kuo J, Lee SL, Kiesler S (2005) Eliciting information from people with a gendered humanoid robot. In: Proceedings of the IEEE international workshop on robot and human interactive communication. IEEE, Nashville. https://doi.org/10.1109/roman.2005.1513773

Qiu L, Benbasat I (2009) Evaluating anthropomorphic product recommendation agents: a social relationship perspective to designing information systems. J Manag Inf Syst 25(4):145-182

Reynolds KE, Beatty SE (1999) Customer benefits and company consequences of customer-salesperson relationships in retailing. J Retail 75(1):11-32

Saygin AP, Chaminade T, Ishiguro H, Driver J, Frith C (2012) The thing that should not be: predictive coding and the uncanny valley in perceiving human and humanoid robot actions. Soc Cogn Affect Neurosci 7(4):413-422

Schuetzler R, Grimes M, Giboney J, Buckman J (2014) Facilitating natural conversational agent interactions: lessons from a deception experiment. In: Proceedings of the international conference on information systems. AIS, Auckland

Shah KB, Shetty MS, Shah DP, Pamnani R (2017) Approaches towards building a banking assistant. Int $\mathrm{J}$ Comput Appl 166(11):1-6

Siegel M, Breazeal C, Norton MI (2009) Persuasive robotics: the influence of robot gender on human behavior. In: IEEE/RSJ international conference on intelligent robots and systems. IEEE, St. Louis, pp 2563-2568. https://doi.org/10.1109/iros.2009. 5354116

Wang W (2017) Smartphones as social actors? Social dispositional factors in assessing anthropomorphism. Comput Hum Behav 68:334-344

Weizenbaum J (1966) ELIZA - a computer program for the study of natural language communication between man and machine. Commun ACM 9(1):36-45

Wen Wan E, Peng Chen R, Jin L (2017) Judging a book by its cover? The effect of anthropomorphism on product attribute processing and consumer preference. J Consum Res 43(6):1008-1030

White RW (1959) Motivation reconsidered: the concept of competence. Psychol Rev 66(5):297

Wilks Y (2010) Is a companion a distinctive kind of relationship with a machine? In: Proceedings of the 2010 workshop on companionable dialogue systems, association for computational linguistics, Stroudsburg, pp 13-18

Zhang T, Zhu B, Lee L, Kaber D (2008) Service robot anthropomorphism and interface design for emotion in human-robot interaction. In: IEEE international conference on automation science and engineering 2008. IEEE, Arlington, pp 674-679 